\title{
Scattering of acoustic phonons in disordered matter: A quantitative evaluation of the effects of positional versus orientational disorder
}

\author{
A. I. Krivchikov, A. N. Yushchenko, V. G. Manzhelii, and O. A. Korolyuk \\ B. Verkin Institute for Low Temperature Physics and Engineering of NAN Ukraine, Kharkov, Ukraine \\ F. J. Bermejo, R. Fernández-Perea, and C. Cabrillo* \\ Instituto de Estructura de la Materia, CSIC and Departamento Electricidad y Electrónica-Unidad Asociada CSIC, \\ Facultad de Ciencia y Tecnología, Universidad del País Vasco/EHU, P.O. Box 644, E-48080-Bilbao, Spain \\ M. A. González \\ Institute Laue Langevin, 6 Rue Jules Horowitz, F-38042 Grenoble Cedex 9, France \\ (Received 14 July 2006; published 9 August 2006)

\begin{abstract}
The thermal conductivity of all three disordered solid phases of ethyl alcohol has been measured. That for the orientationally disordered bcc phase is found to be remarkably close to that for the structurally amorphous solid, especially at low temperatures. The results, which emphasize the role of orientational disorder in phonon scattering, are discussed with the aid of computer simulations on single-crystalline models of both bcc and monoclinic crystals.
\end{abstract}

DOI: 10.1103/PhysRevB.74.060201

PACS number(s): 66.70.+f, 61.43. $-\mathrm{j}, 63.50 .+\mathrm{x}, 65.60 .+\mathrm{a}$

Our current understanding of the mechanisms of heat transport in disordered media rests upon concepts grounded on clean experiments showing that acoustic phonons, especially those having transverse polarization, are the main heat carriers. ${ }^{1}$ Work carried out over the last couple of decades has evidenced striking quantitative similarities in the characteristic thermal conductivity of bulk amorphous materials ${ }^{2}$ between, say, 0.1 and $10 \mathrm{~K}$, independent of chemical composition. Furthermore, such similarity also extends to a good number of disordered crystals, including a quasicrystal, ${ }^{2,3}$ and from the set of collected data it has been inferred that the ratio of the wavelength $\lambda$ of the acoustic wave to the mean free path $l$ of all these solids ranges within $10^{-2}-10^{-3}$, which suggests the presence of "universal" behavior of some sort. On such grounds, it becomes clear that the presence of "glassy dynamics" cannot be attributed in full to the absence of static translational long-range order (LRO).

Some molecular crystals where the individual molecules have random static orientations while their centers of mass are at the nodes of a three-dimensional crystalline lattice are also known to exhibit glasslike excitations. Of those, solid ethyl alcohol is perhaps the most convenient benchmark to carry out a quantitative comparison of the effects caused by the complete lack of $\mathrm{LRO},{ }^{4}$ on the most sensitive property to explore the propagation of excitations in condensed matter, the thermal conductivity. The material, apart from the wellknown monoclinic (fully ordered) crystalline (FOC) modification, can be prepared in three long-lived phases, an amorphous solid or glass, an orientationally disordered crystal (ODC) (or orientational glass) showing static orientational disorder but having translational LRO since the molecules are at the nodes of a bcc lattice, and a crystal with dynamic orientational disorder [rotator-phase crystal (RPC)] which retains LRO as a bcc lattice still exists. Two glass transitions take place about $97 \mathrm{~K}$ between the glass and supercooled liquid and the ODC and RPC. ${ }^{4}$

Here we report on measurements of the thermal conduc- tivity of ethyl alcohol for all the solid phases. The relevance of such an exercise is twofold. First and foremost, as stated in a recent review, ${ }^{2}$ the measurements will provide additional tests on claims of quantitative universality of the properties of heat propagation at low and intermediate temperatures in disordered matter brought forward by a class of materials for which no data were available in Ref. 2. On the other hand, our results may contribute to clarifying the nature of the spectral response observed by means of inelastic x-ray scattering (IXS) on polycrystals of the same material, ${ }^{6}$ for which rather different interpretations have been proposed., 5

The measurements for the three solid phases were carried out under equilibrium vapor pressure at $2-159 \mathrm{~K}$ by the steady-state potentiometric method using a special setup. ${ }^{7}$ The preparation of the glass, disordered crystal, or fully ordered monoclinic phase was carried out following previously described procedures. ${ }^{4}$

Figure 1 illustrates the temperature dependencies of the thermal conductivity $\kappa(T)$ for the glass, orientationally disordered crystal, and fully ordered crystal. Data for the FOC phase exhibit a shape much like that of most crystalline solids explored so far. From melting $\left(T_{m}=159 \mathrm{~K}\right)$ down to some $27 \mathrm{~K}, \kappa(T)$ follows $\kappa(T) \propto \exp (-E / T)$ with $E=38.3 \pm 0.9 \mathrm{~K}$. The maximum is located at $T=12 \mathrm{~K}$ and below $4.5 \mathrm{~K}$ it decreases following $\kappa(T) \propto T^{2}{ }^{8}$ Notice the abrupt decrease in thermal conductivity by $42 \%$ within a narrow temperature interval. Both ODC and glass exhibit a temperature dependence of $\kappa(T)$ characteristic of most amorphous solids. ${ }^{2,9}$ $\kappa(T)$ strongly increases with temperature up to about $5 \mathrm{~K}$. A smeared-out plateau appears within 5-10 K, above which $\kappa(T)$ experiences a further increase. This increase lasts up to $T=50 \mathrm{~K}$, from where $\kappa(T)$ for both samples shows a very mild dependence on temperature up to both glass transition ranges at $T_{g} \simeq 97 \mathrm{~K}$. Data for the ODC closely follow those of the amorphous solid. In real numbers, $\kappa(T)$ for the ODC is higher than that of the structural glass by about $2 \%$ at $2 \mathrm{~K}$, $8 \%$ at $3.2 \mathrm{~K}, 16 \%$ at $10 \mathrm{~K}, 12 \%$ at $25 \mathrm{~K}$, and $13 \%$ above 

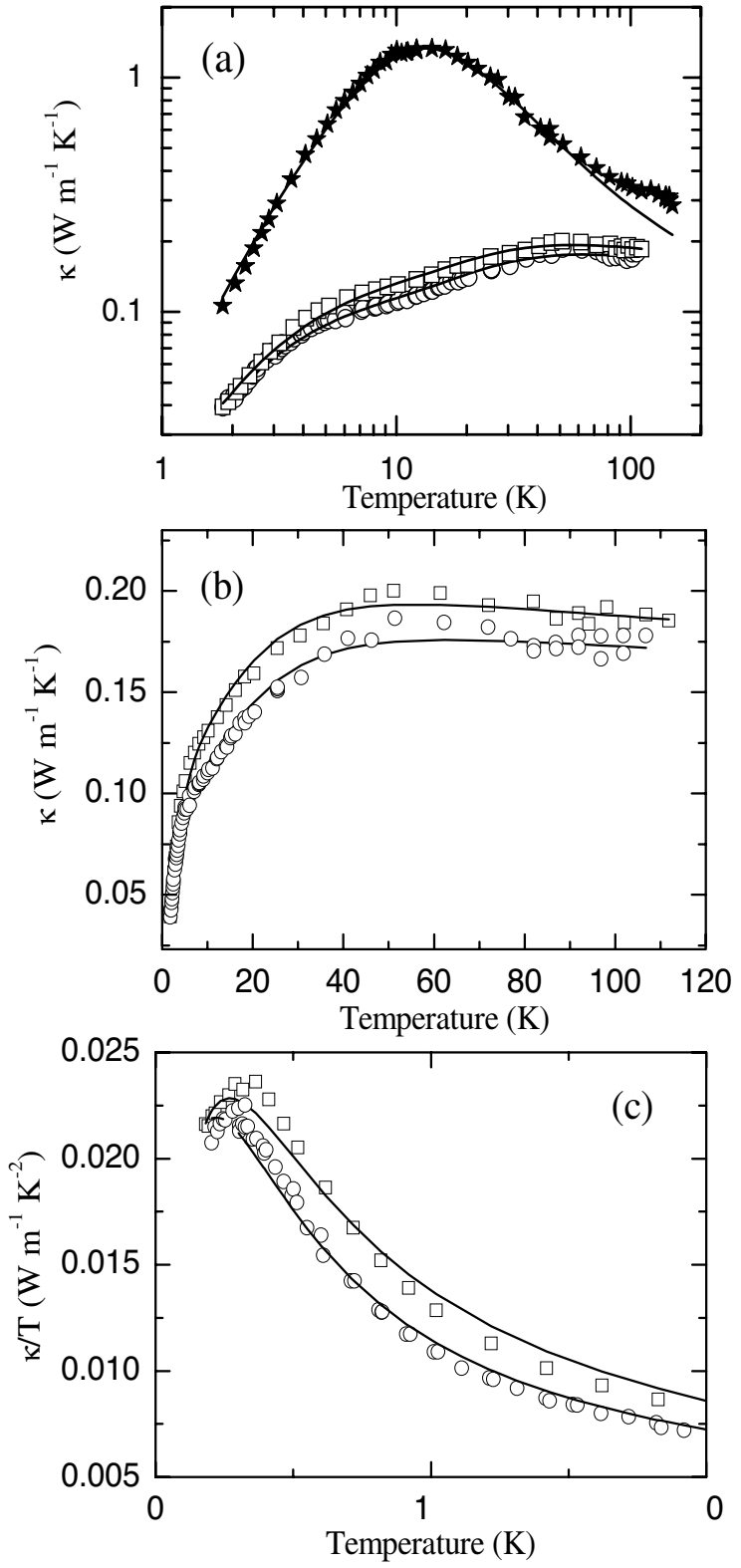

FIG. 1. The top frame depicts the measured thermal conductivity for the FOC (stars), ODC (squares), and glass (circles). Solid lines are model fits as described in the text. The middle frame depicts data for the glass (circles) and ODC (squares) plotted on a linear scale. The bottom frame depicts data in $\kappa / T-T$ coordinates.

$50 \mathrm{~K}$. At temperatures beyond $T_{g}$ our data show that $\kappa(T)$ for the supercooled liquid and for the RPC are both independent of temperature, its value being close to the thermal conductivity of liquid ethanol at the temperature of solidification.

The glasslike behavior of $\kappa(T)$ of both glass and ODC is modeled on phenomenological grounds using the model of soft potentials ${ }^{10-12}$ which portrays phonon scattering as mainly caused by low-energy excitations of a strongly anharmonic ensemble of particles. The scattering rate of acoustic phonons in a disordered system is given by a sum of three terms describing scattering by the tunnel states, classical relaxors, and soft quasiharmonic vibrations, and reads

$$
\begin{aligned}
\tau_{G}^{-1}= & C \pi \omega \tanh \left(\frac{h \omega}{2 k_{B} T}\right)+C \pi \omega\left(\frac{T}{W}\right)^{3 / 4} \ln ^{-1 / 4}\left(1 / \omega \tau_{0}\right) \\
& +\frac{\bar{C} \pi \omega}{6 \sqrt{2}}\left(\frac{\hbar \omega}{W}\right)^{3} .
\end{aligned}
$$

Here $\tau_{0}$ is the inverse of the attempt frequency and is of the order of $10^{-13} \mathrm{~s}$ which along with the sound wave frequencies $(10-100 \mathrm{GHz})$ yields a logarithmic factor $\ln ^{-1 / 4}\left(1 / \omega \tau_{0}\right) \approx 0.7$. The most relevant parameters are the dimensionless $C$ parameter and the characteristic energy $W$. Within the FOC phase, the thermal conductivity is analyzed within the Debye model in terms of a total relaxation time $\tau_{\text {total }}$ given as a sum of relaxation rates $\tau_{\text {total }}^{-1}=\tau_{U}^{-1}+\tau_{B}^{-1}+\tau_{G}^{-1}$ for umklapp processes $\tau_{U}$ [important for temperatures beyond the maximum of $\kappa(T)$ ], boundary scattering $\tau_{B}$, and scattering leading to the glasslike thermal conductivity, $\tau_{G}$. Here, $\tau_{U}^{-1}=B \omega^{2} T \exp (-E / T)$ for the umklapp process and $\tau_{B}^{-1}=v / L$ for the boundary scattering, where $L$ stands for the crystallite size. Also, $\tau_{\text {total }} \geqslant \tau_{\min }=2 / \omega{ }^{9}{ }^{9}$ The experimental results together with the calculated curves are shown in Fig. 1. The derived parameters yield values for the Debye temperature $\Theta_{D}$ of $110 \mathrm{~K}$ for the glass, $109 \mathrm{~K}$ for the ODC, and $116 \mathrm{~K}$ for the FOC that come close to those obtained from the low-temperature heat capacity. ${ }^{4}$ Also, values for the strength factor $C$ yield $8.0 \times 10^{-4}$ for the glass and 8.6 $\times 10^{-4}$ for the ODC while $W$, which characterizes the crossover from a regime dominated by phonon scattering by lowenergy excitations (tunneling states and classical relaxors) into one of scattering by soft quasiharmonic vibrations, yields $3.6 \mathrm{~K}$ for the glass and $4.0 \mathrm{~K}$ for the ODC.

Our data for both glassy phases show that their heat conductivities are strikingly close to each other and greatly differ from those for the FOC. Put into different words, the analysis here pursued shows, whatever the nature of the microscopic entities executing motions able to scatter phonons, they share basically the same properties in the fully amorphous and ODC phases.

From the relationship given by Pohl et $a .^{2}$ a value for the ratio of the wavelength of the acoustic wave $\lambda$ to its mean free path $l$ is derived,

$$
\frac{\lambda}{l}=\pi^{2} C,
$$

and yields values $\simeq 8 \times 10^{-3}$, which are in quantitative agreement with data reported in Ref. 2 and thus lend further support to the claim of universal behavior there expounded, since it is shown to be also followed by this class of materials.

The present results are in agreement with Brillouin lightscattering data from Criado et al. ${ }^{4}$ which show ratios of the Brillouin linewidth to the peak frequency (i.e., a measure of sound attenuation) having close values for both ODC and glass and increasing with temperature up to $\approx 60 \mathrm{~K}$. However, our data are in contrast with findings pertaining to probes of higher frequencies typically employed in IXS or in elastic neutron scattering (INS) experiments such as those reported in Ref. 6. There, the wave-vector dependence of the 
frequency and linewidth of the inelastic signal for all the three phases at $T=86 \mathrm{~K}$ is found to be very similar, from where inferences about a common origin of excitations in all phases are derived. Data shown in Fig. 1, however, show that at such a temperature $\kappa(T)$ for the FOC is about twice that of the glass. To clarify the origin of this discrepancy we have profited from the presence within the ODC of an underlying bcc crystal lattice. This enables us to track down the dispersion relation for acoustic phonons throughout reciprocal space, an issue that cannot be pursued on polycrystalline samples. ${ }^{13}$

As a point of departure we will here consider the lattice dynamics model for the FOC, ${ }^{14}$ which was scrutinized against the experimentally derived vibrational densities of states and found to provide an adequate representation of the harmonic crystal dynamics up to about $25 \mathrm{meV}$. Calculated phonon dispersion branches along the highest-crystalsymmetry directions show that acoustic phonons are bounded below $\approx 8 \mathrm{meV}$. Above that limit, acoustic phonons are strongly hybridized with a large number of vibrations of optic type and therefore their frequency-wave-vector behavior can only be disentangled by careful measurements on single crystals.

Because of the difficulty in preparing large single crystals for both FOC and ODC phases, we have made recourse to molecular dynamics (MD) simulations on models of both ODC and FOC single crystals using for the purpose potentials and algorithms already tested against experimental data. ${ }^{15}$ The adequacy of classical tools such as those provided by MD methods to explore properties at low temperatures needs to be established and account taken of unavoidable quantum effects. The strength of those is usually assessed by comparison of the de Broglie thermal wavelength $\lambda_{T}=h / \sqrt{2 \pi M k_{B} T}$ to the mean particle separation $d_{\text {min }}$. Quantum interference effects are deemed to be significant only if $\lambda_{T}>d_{\text {min }}$. Since we are here interested in the behavior of acoustic phonons, the relevant mass is the molecular mass $M \approx 46$ amu and $d_{\text {min }} \approx 5 \AA$, as determined from the first intermolecular peak in the experimental radial distributions. ${ }^{15}$ At $T=5 \mathrm{~K} \lambda_{T} \approx 1 \AA$ which ensures that results pertaining to whole-molecule motions are not expected to exhibit large quantum behaviors. On the other hand, the strength of anharmonic effects was previously measured from the temperature (see Criado et al. ${ }^{4}$ ) and volume (see Fischer et al. ${ }^{4}$ ) dependencies of the phonon frequencies. This provides quantitative estimates of the importance of such effects, which amount to a frequency softening of about $3 \%$ at low temperatures up to about $10 \%$ at $T=90 \mathrm{~K}$.

The calculated dynamic structure factors along the main (001, 010, and 100) crystal directions and temperatures comprising $5-30 \mathrm{~K}$ were analyzed in detail. A sample of the results is shown in Fig. 2. The lowest temperature $(5 \mathrm{~K})$ corresponds to the upper limit of the strong rise with temperature of $\kappa(T) ; T=30 \mathrm{~K}$ is the limiting temperature where the Debye model is able to account for the measured specific heat ${ }^{14}$ or put into different words, where the specific heat can be fully accounted for in terms of acoustic excitations only. Finally, $T=100 \mathrm{~K}$ comes close to the stability limit of the ODC and also to the actual temperature where IXS measurements were conducted.

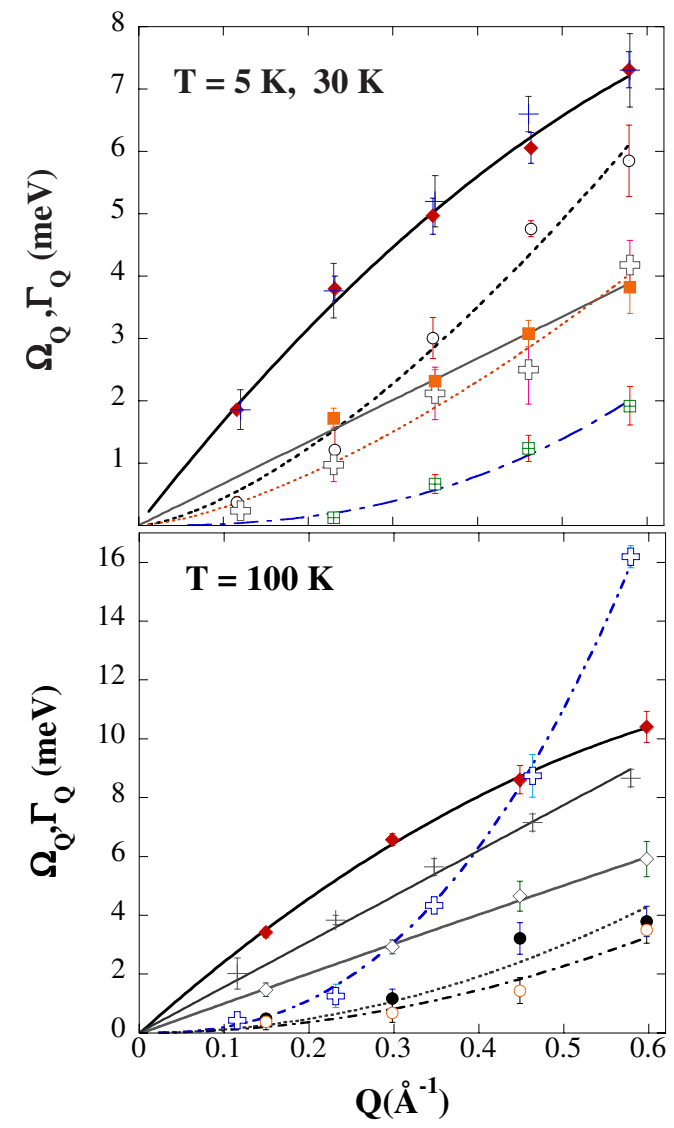

FIG. 2. (Color online) Calculated phonon frequencies $\Omega_{Q}$ and phonon linewidths $\Gamma_{Q}$ along the (001) crystal directions. The upper frame compares data for the orientationally disordered crystal at low $(5 \mathrm{~K})$ and moderate $(30 \mathrm{~K})$ temperatures. Thin and open crosses display $\Omega_{Q}$ and $\Gamma_{Q}$ for the longitudinal phonon at $T=5 \mathrm{~K}$. Full and open squares with a cross depict frequencies and damping for the transverse excitation sampled at the same temperature. Filled lozenges and open circles with a dot show dispersion frequencies and damping terms for the longitudinal phonon at $30 \mathrm{~K}$. The lower frame compares data for the FOC and ODC crystals at high temperature $(100 \mathrm{~K})$. Longitudinal and transverse phonon frequencies for the FOC are shown by filled and open lozenges, respectively. The corresponding linewidths are depicted by filled and open circles. Data for the frequencies and widths of the ODC crystal at the same temperature shown by crosses and open crosses, respectively. Lines drawn through the points are approximations given in terms of simple power laws (see text).

Our results for the glassy crystal show that for temperatures above $5 \mathrm{~K}$ only the longitudinal acoustic phonon survives the strong scattering processes induced by orientational disorder. The graphs shown in the upper frame of Fig. 2 for the lowest temperature depict a longitudinal branch that corresponds to a hydrodynamic sound velocity of $2639 \mathrm{~m} \mathrm{~s}^{-1}$ that remains as a well-defined peak throughout the Brillouin zone. Damping of such excitation follows a subquadratic dependence with wave vector, the linewidth being well approximated by $\Gamma_{Q}=9.8 Q^{3 / 2} \AA^{-3 / 2}$. A transverse excitation also appears within the first two Brillouin zones. Its linear dispersion corersponds to a propagation velocity of $989 \mathrm{~m} \mathrm{~s}^{-1}$ while its large damping is accounted by a stronger 
than quadratic dependence $\Gamma_{Q}=7.9 Q^{5 / 2} \AA^{-5 / 2}$. Increasing the temperature well above the plateau in $\kappa(T)$ has little effect on the frequencies of the longitudinal phonon while its damping increases by up to $\Gamma_{Q}=13.9 Q^{3 / 2} \AA^{-3 / 2}$. The absence of a transverse acoustic branch for such a temperature is here ascribed to scattering from static orientational disorder since, as shown below, such phonons are observable in the hightemperature crystal where low-energy librations are thermally populated. Increasing again the temperature up to a value consistent with that explored experimentally, that is, $0.88 T_{g}$, leads to a substantial broadening of the longitudinal mode frequencies for the ODC. These, as shown in the lower frame of Fig. 2 correspond to a hydrodynamic value for the sound velocity of $2349 \mathrm{~m} \mathrm{~s}^{-1}$, a result in agreement with light-scattering results. ${ }^{4}$ In addition, the phonon remains as a well-defined entity up to wave vectors corresponding to onehalf of the Brillouin zone only, and the damping coefficient can be accounted for by $\Gamma_{Q}=62.40 Q^{5 / 2}$. Data for the monoclinic crystal also depicted in Fig. 2 show a longitudinal branch with a hydrodynamic limiting value of $3917 \mathrm{~m} \mathrm{~s}^{-1}$ together with a transverse mode propagating with a hydrodynamic velocity of $1574 \mathrm{~m} \mathrm{~s}^{-1}$. Damping of both modes is well accounted for by hydrodynamic $Q$ dependences with coefficients of 11.98 and $9.10 \AA^{2} \mathrm{meV}$, respectively.

The present data contrast with those derived from an analysis of IXS spectra of polycrystals that considers all inelastic intensities up to $\approx 25 \mathrm{meV}$ arising from a single longitudinal mode. ${ }^{6}$ Particularly striking are the large damping terms here found for the ODC, which witness a strong reduction in the phonon mean free path at high temperature for momentum transfers below some $0.4 \AA^{-1}$. The results of Ref. 6 portray excitation linewidths for both FOC and ODC attaining roughly the same values, and on such grounds it is inferred that structural disorder plays a small role. Here we show that thermal disorder significantly shortens the lifetimes of acoustic excitations within the ODC, which leads to significantly smaller heat conductivities, in agreement with experiment. To reconcile both results it is worth recalling that IXS (or INS) experiments carried out on polycrystals ${ }^{5}$ mostly sample inhomogeneous spectra composed of excitations additional to those of acoustic character,${ }^{14}$ which pre- empts the interpretation of maxima and linewidths of the spectral bands in terms of physical frequencies and their damping terms.

From the data and arguments described in the above paragraph some inferences about the nature of excitations in glassy crystals are derived which, on the basis of the proximity of its thermal transport behavior to that of the structural glass should also apply to the amorphous solid. First, transverse phonons which are deemed to be the main heat carriers at low temperatures are strongly scattered by disorder at temperatures (frequencies) about the plateau in $\kappa(T)$. Such modes, however, remain well-defined entities in the high-temperature FOC despite its substantial anharmonicity ${ }^{4}$ and thus explain the higher conductivity of the ordered crystal over that shown by the ODC at high temperature. Second, our simulation results suggest that propagation of longitudinal excitations in the ODC at low temperatures takes place beyond frequencies characteristic of the "boson peak" (BP) which for this material appears at $\approx 2 \mathrm{meV} .{ }^{4,15}$ Such frequencies are, however, confined to values not far in excess of that of the BP at high temperatures. As far as identification of the prime entities responsible for scattering of acoustic phonons, low-energy torsional librations about the $\mathrm{C}-\mathrm{C}$ bond $^{14}$ are deemed to be the main contributors to such processes. As shown previously for other materials ${ }^{16}$ scattering from such excitations leads to a plateau in $\kappa(T)$ and also reproduces the observed trends up to relatively high temperatures.

In summary, measurements of $\kappa(T)$ show the close analogy of heat propagation processes in materials having an underlying crystal lattice but orientational disorder and fully amorphous solids. The results show that orientational disorder accounts for the perhaps most emblematic property of the glassy state, the thermal conductivity, at low and intermediate temperatures. Our findings yield additional support to claims of quantitative universal behavior for this quantity for an ample set of disordered materials. ${ }^{2}$ Results from molecular dynamics simulations on the single-crystal models provide a microscopic view of the heat-carrying processes. These emphasize the role of both static and thermal disorder as limiting factors for heat transport.

\footnotetext{
*Electronic address: jbermejo@we.lc.edu.es

${ }^{1}$ P. D. Wu et al., J. Low Temp. Phys. 113, 123 (1998).

${ }^{2}$ R. O. Pohl et al., Rev. Mod. Phys. 74, 991 (2002).

${ }^{3}$ M. Kobas et al., Phys. Rev. B 71, 224206 (2005).

${ }^{4}$ M. A. Ramos et al., Phys. Rev. Lett. 78, 82 (1997); A. Criado et al., Phys. Rev. B 61, 8778 (2000); M. Jimenez-Ruiz et al., Phys. Rev. Lett. 83, 2757 (1999). C. Cabrillo et al., Phys. Rev. B 64, 064206 (2001); H. E. Fischer et al., Phys. Rev. Lett. 82, 1193 (1999); M. A. Miller et al., Phys. Rev. B 57, R13977 (1998).

${ }^{5}$ B. Rufflé et al., Phys. Rev. Lett. 96, 045502 (2006).

${ }^{6}$ A. Matic et al., Phys. Rev. Lett. 93, 145502 (2004).

${ }^{7}$ A. I. Krivchikov et al., J. Low Temp. Phys. 139, 693 (2005); A.
}

I. Krivchikov et al., Phys. Chem. Chem. Phys. 7, 728 (2005).

${ }^{8}$ P. Stachowiak et al., Phys. Rev. B 69, 024305 (2004); 67, 172102 (2003).

${ }^{9}$ D. G. Cahill et al., Phys. Rev. B 46, 6131 (1992).

${ }^{10}$ U. Buchenau et al., Phys. Rev. B 46, 2798 (1992).

${ }^{11}$ M. A. Ramos et al., Phys. Rev. B 55, 5749 (1997).

${ }^{12}$ F. J. Bermejo et al., J. Low Temp. Phys. 139, 567 (2005).

${ }^{13}$ F. W. de Wette and A. Rahman, Phys. Rev. 176, 784 (1968).

${ }^{14}$ C. Talón et al., Phys. Rev. B 58, 745 (1998).

${ }^{15}$ M. A. González et al., Phys. Rev. B 61, 6654 (2000); Phys. Rev. E 61, 3884 (2000).

${ }^{16}$ F. J. Bermejo et al., Phys. Rev. B 49, 8689 (1994). 